\title{
Kinetics of Hydrogen Storage on Catalytically-Modified Porous Silicon
}

\author{
Mawla Boaks ${ }^{1}$ and Peter J. Schubert ${ }^{2}$
}

\begin{abstract}
Porous silicon has been demonstrated as a hydrogen storage media with surface-bound hydrogen content as high as $6.6 \%$ by weight. Hydrogenated porous silicon is readily synthesized by electrochemical etching in a solution of hydrofluoric acid. Hydrogen gas can be released thermally at temperatures starting at $280 \mathrm{C}$. It has been proposed that a suitable catalyst at the pore mouth can both reduce the desorption temperature and facilitate gaseous recharge of the silicon matrix. This work presents a detailed kinetic study using density functional theory (DFT) of a reversible hydrogen storage system based on porous silicon via the mechanisms of dissociation, spillover, and bond-hopping of hydrogen atoms. For each of these steps, activation energy values and vibrational frequency has been determined. Using these activation energies along with vibrational frequency values evaluated from the micro level DFT study, the kinetic performance of catalyticallymodified porous silicon as a potential hydrogen storage material has been completed for the first time. The energy difference between full and empty charge is computed at the atomic scale and compared to macroscopic calculations, showing close agreement. These results show the potential for rapid recharge at 8 bar at temperatures commensurate with waste heat from a proton-exchange membrane fuel cell.
\end{abstract}

\section{INTRODUCTION}

Efficient and reversible storage is the primary challenge to a Hydrogen Economy. Hydrogen is 6.9 times lighter than Lithium per unit electric charge providing a fundamental advantage over electrochemical battery technology. Of course hydrogen storage must be paired with a fuel cell to compare directly to a battery, so the ultimate theoretical advantage over lithium metal batteries will be moderated, perhaps an advantage of a factor of 3 .

Ultra-high pressure storage of hydrogen in gaseous form incurs a heavy penalty in parasitic energy loss needed for compression. Widespread gaseous use implies large central hydrogen generation plants with vast networks of specialized pipelines. The enormous capital expense of such infrastructure, needed in advance of market adoption of hydrogenfueled vehicles and home fuel cell applications presents a dilemma from which there is no obvious emergence. Cryogenic storage in liquid form requires energy-intensive refrigeration and substantial insulation to reduce boil-off. While useful for large rockets, liquid storage is impractical for vehicles or for diffuse distribution. Solid state storage in metal hydrides is handicapped by the mass of the metals required, and by the highly exothermic nature of the recharge

\footnotetext{
${ }^{1} \mathrm{M}$. Boaks graduated with a MS degree from IUPUI and currently pursuing Ph.D. at Electrical and Computer Engineering Department, Brigham Young University, Provo, UT 84602, USA. mboaks @byu . edu

${ }^{2} \mathrm{P}$. J. Schubert is a professor at the Department of Electrical and Computer Engineering, Indiana University-Purdue University Indianapolis, Indianapolis, IN 46202, USA pjschube@iupui.edu
}

process demanding either slow absorption or complex cooling mechanisms.

A novel method of solid state hydrogen storage using porous silicon has been proposed and studied [1], and is now the subject of four US patents. Silicon bonds with hydrogen atoms at energies lower than does carbon, and hydrogen has been shown to bond-hop along silicon surfaces. Porous silicon can be synthesized with very high surface area. The greatest energy barrier in such a system is the dissociation of gaseous dihydrogen $\left(\mathrm{H}_{2}\right)$ into atomic hydrogen, which can be mediated by the introduction of a catalyst. Strategic placement of a catalyst such as palladium at pore mouths provides a reversible pathway from dihydrogen gas to dissociated hydrogen on clusters of catalyst which transfers to the silicon surface via spillover and then bond-hops along the surface to form a solid state hydrogen storage reservoir. This system is charged by gas pressure and discharged by temperature. An additional discharge mechanism is available via infra red light which passes through lightly-doped silicon but is absorbed by the Si-H bond.

Herein the kinetics of this reaction pathway are studied using Density Functional Theory (DFT). In this way the energy barriers for each of the three steps can be studied individually, namely: dissociation, spillover, and bond-hopping. In the composite the charging rate of catalytically-modified porous silicon can be estimated. These detailed calculations are compared with first-order macroscopic kinetic calculations to provide support for the feasibility of the results.

\section{METHODS}

\section{A. Chemical Kinetics}

In this proposed storage system gaseous hydrogen goes through a series of equilibrium reactions that form different types of compounds that bond silicon and hydrogen. At the beginning, molecular hydrogen (dihydrogen) is dissociated into atomic hydrogen by the palladium catalyst.

$$
H_{2} \rightarrow 2 H_{P d}^{+}
$$

These hydrogen ions are mobile and can spillover onto the dangling or vacant bonds available on the silicon surface, creating the $\mathrm{Si}-\mathrm{H}$.

$$
H_{P d}^{+}+S i^{*} \rightarrow S i H
$$

The net chemical reaction, mediated by the catalyst, is as follows:

$$
\mathrm{H}_{2}+2 \mathrm{Si}^{*} \rightarrow 2 \mathrm{SiH}
$$


From the neighborhood of a catalyst atom or cluster those hydrogen atoms which have moved onto the silicon surface by spillover can migrate across the surface by bondhopping. Such surface diffusion is treated in series with the combination of dissociation and spillover. Dissociation from gaseous dihydrogen is modeled to first order by mass transfer according to:

$$
-r_{d}=k_{b}\left(C_{b}-2 C_{i}\right)
$$

Here, $r_{d}, k_{b}, C_{b}, C_{i}$ represent mass transfer rate by diffusion from gas to surface, mass transfer coefficient from the bulk dihydrogen gas, concentration of hydrogen in the bulk gas and atomic hydrogen concentration at interface with the catalyst, respectively. The first order reaction from the heterogeneous catalyst to the silicon is governed by:

$$
-r_{v}=k_{i} C_{i}
$$

Here, $r_{v}, k_{i}$, and $C_{i}$ are spillover reaction rate, rate constant, and the concentration of $H$ respectively. During steady state charging the reaction rate of dissociation and spillover are equal so $-r_{d}=-r_{v}$, thus:

$$
k_{b}\left(C_{b}-2 C_{i}\right)=k_{i} C_{i}
$$

After simplifying it becomes:

$$
C_{i}=\frac{k_{b}}{k_{i}+2 k_{b}} C_{b}
$$

The overall rate, driven by the concentration and hence pressure of the recharging molecular hydrogen gas will be given by:

$$
-\bar{r}=\frac{k_{i} k_{b}}{k_{i}+2 k_{b}} C_{b}
$$

In general, rate constant is given by:

$$
k=\nu * \exp \left(-\frac{E_{a}}{R T}\right)
$$

Here, $\nu$ is the vibrational frequency and $E_{a}$ is the activation energy. Both of these quantities are evaluated using DFT for hydrogen dissociation, spillover, and bond hopping process.

\section{B. Nanoporous Silicon Matrix}

Silicon has a diamond cubic crystal structure with a lattice constant of $5.43 \AA$. The conventional silicon unit cell consists of 8 atoms. In our nanoporous silicon matrix, $3.5 \mathrm{~nm}$ pore diameter is considered based on experimental evidence. This requires at least 8 unit cells in both $\mathrm{x}$ and $\mathrm{y}$ directions to allocate a complete pore. Four-fold symmetry was used to reduce computational effort. A silicon supercell consisting of $4 \times 4 \times 4$ conventional unit cells was constructed. All the atoms lying within the pore radius of $1.75 \mathrm{~nm}$ were removed to imitate the electrochemical etching procedure done in laboratory to produce nanoporous silicon. The pore depth was extended all the way to the bottom of the supercell. This resulted in a hexagonal shape pore with edges along (100) and (110) planes.

All the DFT computations were done in open source DFT tool ABINIT. ABINIT default LDA: new Teter (4/93)

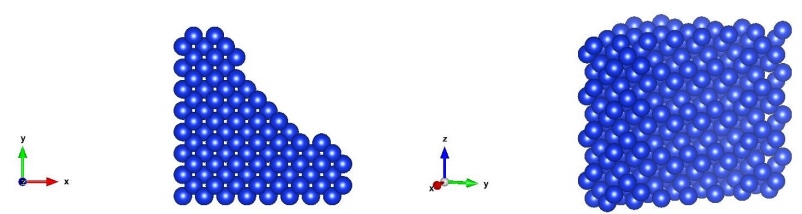

Fig. 1. Nanoporous silicon matrix accommodating one quarter of a pore in a $4 \times 4 \times 4$ silicon supercell: top view (left) and side view (right).

with spin-polarized option was used as exchange-correlation functional throughout our DFT computation. In ABINIT, periodic boundary condition is applied in all three dimensions that creates interaction between electron densities at the periodic boundaries. Literature survey suggested a vacuum space of $15 \AA[3]$ to eliminate interaction between periodic images. Therefore, the system was expanded by another 4 conventional unit cells along $\mathrm{z}$-dimension whereas $\mathrm{x}$ and $\mathrm{y}$ dimensions were kept same as before resulting in a $4 \times 4 \times 8$ silicon supercell. In this newly formed supercell, the upper half was kept empty and there were only atoms in lower half of the supercell along z-direction. The $1^{\text {st }}$ Brillouin zone was initially integrated by $2 \times 2 \times 2$ Monkhorst-Pack grid. The introduction of vacuum corresponds to a decrease in reciprocal space by a factor of 2 . Number of k-points along z-dimension was reduced accordingly resulting in $2 \times 2 \times$ 1 Monkhorst-Pack grid. Troullier-Martins pseudopotentials were used for all the elements in our system. A cutoff energy of $435 \mathrm{eV}$ was used for all the DFT computations. Tolerance value of $0.00002 \mathrm{eV}$ was used. All the computations were carried out in a fast research supercomputer called Big Red II. KGB parallelization was used to further increase the computational speed where standard data partitioning was done over processors corresponding to k-points, block of bands, as well as Fourier space of coefficients over plane wave basis set[4].

\section{Vibrational Frequency}

Since the hydrogen atom is considered to be vibrating on the silicon surface, the interaction between hydrogen atom and silicon atoms may impact the associated vibrational modes. Let us consider $\mathrm{N}$ atoms in the system and their Cartesian coordinates are defined as a single vector, $\mathrm{r}=$ $\left(r_{1}, \ldots ., r_{3 N}\right)$. We further consider the local energy minima is located at $r_{0}$. New coordinate is defined as $x=r-r_{0}$. Taking the Taylor expansion along the energy minima at $r_{0}$ ignoring terms with order greater than 2 yields:

$$
E=E_{0}+\frac{1}{2} \sum_{i=1}^{3 N} \sum_{j=1}^{3 N}\left[\frac{\partial^{2} E}{\partial x_{i} \partial x_{j}}\right]_{x=0} x_{i} x_{j}
$$

The first derivative term in Taylor expansion is zero because it was evaluated at the energy minimum. Let us consider a Hessian matrix consisting of $3 N \times 3 N$ elements defined by,

$$
H_{i j}=\left[\frac{\partial^{2} E}{\partial x_{i} \partial x_{j}}\right]_{x=0}
$$


If we define the atoms as classical particles that follows Newton's law of motion, then we will have, $F_{i}=m_{i}$ $\left(d^{2} x_{i} / d t^{2}\right)$. Here, the force and mass associated with $i$ th coordinate are $F_{i}$ and $m_{i}$ respectively and $\left(d^{2} x_{i} / d t^{2}\right)$ is the acceleration of this coordinate.Rewriting the equation of motion in matrix form gives:

$$
\frac{d^{2} \mathbf{x}}{d t^{2}}=-\mathbf{A} \mathbf{x}
$$

This is the mass-weighted Hessian matrix whose elements are defined as, $A_{i j}=H_{i j} / m_{i}$. This matrix form equation has a special set of solutions associated with the eigenvectors of A called the normal modes. The eigenvector of this matrix and their eigenvalues satisfy following equation,

$$
\mathbf{A e}=\lambda \mathbf{e}
$$

Here, $\lambda$ and $\mathbf{e}$ are eigenvalues and eigenvectors of matrix A respectively. In general, $\mathbf{A}$ has $3 \mathrm{~N}$ eigen vectors and corresponding eigenvalues. The $3 \mathrm{~N}$ normal mode frequencies then can be defined using the $3 \mathrm{~N}$ eigenvalues as follows,

$$
\nu_{i}=\frac{\sqrt{\lambda_{i}}}{2 \pi}
$$

Now, the frequency calculation of hydrogen atom will result in $3 \mathrm{~N}=3$ eigenvalues due to the fact that hydrogen atom has 3 (three) degrees of freedom along three spatial coordinates. The Hessian matrix in our case will be a $3 \times 3$ matrix defined as follows:

$$
H_{i j}=\left(\begin{array}{ccc}
\frac{\delta^{2} E}{\delta x^{2}} & \frac{\delta^{2} E}{\delta x \delta y} & \frac{\delta^{2} E}{\delta x \delta z} \\
\frac{\delta^{2} E}{\delta y \delta x} & \frac{\delta^{2} E}{\delta y^{2}} & \frac{\delta^{2} E}{\delta y \delta z} \\
\frac{\delta^{2} E}{\delta z \delta x} & \frac{\delta^{2} E}{\delta z \delta y} & \frac{\delta^{2} E}{\delta z^{2}}
\end{array}\right)
$$

From finite difference approximation the diagonal elements of the Hessian matrix are defined as, for example:

$$
\frac{\delta^{2} E}{\delta x^{2}} \approx \frac{E(x+\delta x, y, z)-2 E(x, y, z)+E(x-\delta x, y, z)}{\delta x^{2}}
$$

However, the non-diagonal elements are defined as following according to finite difference approximation:

$\frac{\delta^{2} E}{\delta x \delta y} \approx \frac{E(x+\delta x, y+\delta y, z)-E(x+\delta x, y-\delta y, z)-E(x-\delta x, y+\delta y, z)+E(x-\delta x, y-\delta y, z)}{4 \delta x \delta y} ;$

But since the $\mathrm{H}$ atom movement is along the (110) plane, we need to rotate the $\mathrm{x}$ and $\mathrm{y}$ axis by an angle of $\theta=45$ degrees. The following rotation matrix is used to serve the purpose:

$$
R=\left(\begin{array}{cc}
\cos \theta & -\sin \theta \\
\sin \theta & \cos \theta
\end{array}\right)
$$

The elements of the Hessian matrix were modified accordingly. It is worth mentioning that all the terms present in the numerator at the right hand side of above equations are evaluated using DFT. The mass-weighted Hessian matrix A will have 3 eigenvectors $e_{1}, e_{2}$, and $e_{3}$ and corresponding 3 eigenvalues $\lambda_{1}, \lambda_{2}$, and $\lambda_{3}$. For these eigenvalues, corresponding normal mode frequencies will be calculated using equation (3). Discrete distances used in equations (5) and (6) were computed with movements about the energy minimum of $0.04 \AA$. Finally, when electron-rich catalyst atoms were included, those silicon atoms more than 4 unit cells remote from the pore wall were omitted in order to reduce system memory.

\section{RESULTS}

\section{A. Catalyst}

In as-synthesized nanoporous silicon (npSi) based solid state hydrogen storage systems, palladium is used as catalyst for its ability to reduce the substantial gaesous dihydrogen $\left(\mathrm{H}_{2}\right)$ dissociation energy barrier. In this npSi, $\mathrm{Si}-\mathrm{H}$ and $\mathrm{Si}-$ $\mathrm{H}_{2}$ are most prominent, with the presence of a small number of $\mathrm{Si}-\mathrm{H}_{3}$ bonds[5] where the silicon atom is bonded to only one neighboring silicon atom and are therefore the structurally weakest spot. These weak spots are supposed to give away dihydrogen gas first during dehydrogenation[6]. In this catalyst deposition method, storage media is initially heated to a temperature for partial dehydrogenation where Si$\mathrm{H}_{3}$ bonds give away two hydrogen atoms. The nanoporous silicon matrix is then cooled from this temperature and $\mathrm{Pd}$ catalyst atom is deposited in a solution[7]. Catalyst atoms in clusters of at two or more atoms are expected to facilitate gaseous hydrogen dissociation and the spillover process[8]. As the size of the cluster increases, multiple $\mathrm{H}_{2}$ molecules are adsorbed to the cluster[10]. For palladium, the tetrahedral structure consisting of 4 (four) atoms is found to be lowest in energy in a prior DFT study[9] and thus most stable. Therefore, we have evaluated this tetrahedron structure of catalyst atoms at three different locations as possible strategic locations for catalysis. Palladium clusters were placed on and adjacent to the intersection of (100) and (110) planes at the pore edge near the pore mouth. These locations are depicted in figures 2, 3, and 4. Dark blue spheres represent silicon atoms in a nanoporous silicon matrix whereas silver balls represent $\mathrm{Pd}$ catalyst atoms deposited at strategic location.

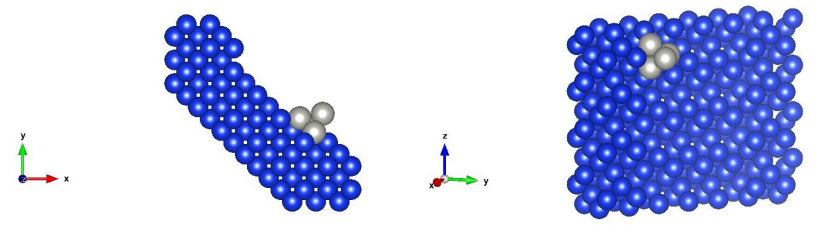

Fig. 2. Cluster of $4 \mathrm{Pd}$ atoms placed on a quarter pore of porous silicon adjacent to the intersection of (100) and (110) plane: top view (left) and side view (right).
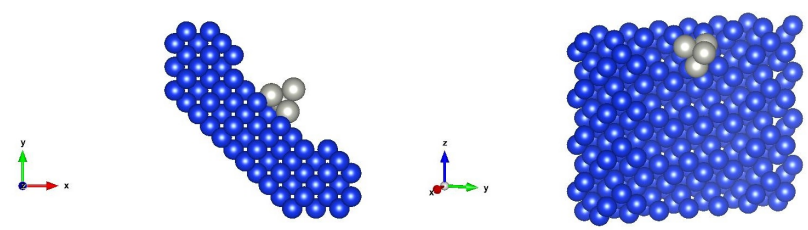

Fig. 3. Cluster of $4 \mathrm{Pd}$ atoms placed on a quarter pore of porous silicon along (110) plane: top view (left) and side view (right). 

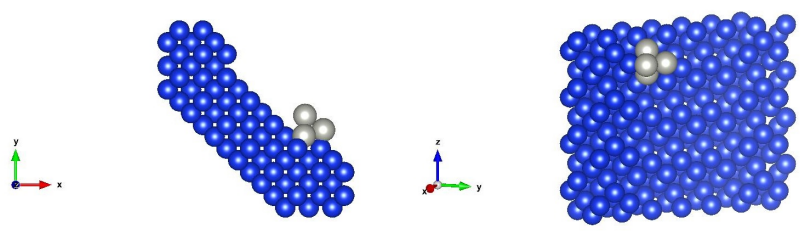

Fig. 4. Cluster of $4 \mathrm{Pd}$ atoms placed on a quarter pore of porous silicon at the intersection of (100) and (110) plane: top view (left) and side view (right).

Two different Pd-Pd bond lengths of 2.60 and 2.69 Angstrom have been reported for a Pd tetrahedron structure supported on a substrate[9]. Therefore, we computed ground state total energy for Pd clusters located at the three proposed locations for bond lengths of both 2.60 and $2.69 \AA$. Naturally, the location with lowest ground state total energy represents the most stable location. In our DFT calculations, a Pd tetrahedron cluster with a Pd-Pd bond length of 2.69 $\AA$ located adjacent to the intersection of (100) and (110) plane was found to be the most stable location. Subsequent dissociation and spillover studies were performed with this site as the strategic location for the 4-atom Pd catalyst cluster.

\section{B. Dissociation}

In a study of dissociation, the silicon supercell was defined with vacuum volume along the $\mathrm{z}$-coordinate, representing the gaseous headspace above the porous silicon surface. A single gaseous $H_{2}$ was sited $15 \AA$ above from the surface, and centered above the pore void. The location of the $\mathrm{H}_{2}$ molecule was positioned such that its wave function does not overlap with the silicon atoms, thus ensuring sufficient separation between periodic images to facilitate more accurate plane wave solutions[3]. Moc et al. have demonstrated that when $\mathrm{H}_{2}$ is dissociated on a $\mathrm{Pd}_{4}$ cluster the individual hydrogen atoms bridge those Pd-Pd edges on opposing sides of the tetrahedron[12]. We configured $\mathrm{Pd}_{4} \mathrm{H}_{2}$ structures such that the dissociated $\mathrm{H}$ atoms are placed at equal distances of $1.66 \AA$ from the Pd atoms. There are two such Pd-Pd edges which do not share any Pd atom. For our nanoporous silicon matrix, three such configurations were studied. In two of them, the dissociated hydrogen atom gets overlaps a $\mathrm{Si}-\mathrm{H}$ bond length and were discarded leaving one configuration which is illustrated in figure 5, where the hydrogen atoms are shown as small red spheres.
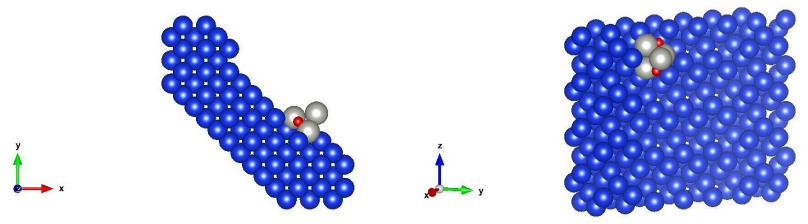

Fig. 5. $H_{2}$ dissociation on Pd tetrahedron cluster: top view (left) and side view (right).

The ground state total energy was computed for before and after hydrogen dissociation phase. The difference between these two resulted in a dissociation energy barrier of 0.47 $\mathrm{eV}$, a result which is significantly lower than previous work reported by the researchers.

\section{Spillover}

Hydrogen uptake in a storage medium is significantly increased by spillover from supported heterogeneous metal catalyst[8][11] where dissociated hydrogen atoms move from the catalyst to nearby low valence silicon surface atoms. Because the Pd atoms forming the tetrahedron lands on some of the lower coordination silicon atoms at the surface, there were silicon atoms with coordination number 2 and 3 available for spillover within a close proximity to the dissociated hydrogen atoms. Among the three available silicon atoms, two had coordination number 2 whereas the other atom had a coordination number of 3. Figures 6 and 7 demonstrate the state of the matrix after hydrogen spillover to these two silicon atoms respectively. Corresponding ground state total energy for both was computed using DFT.

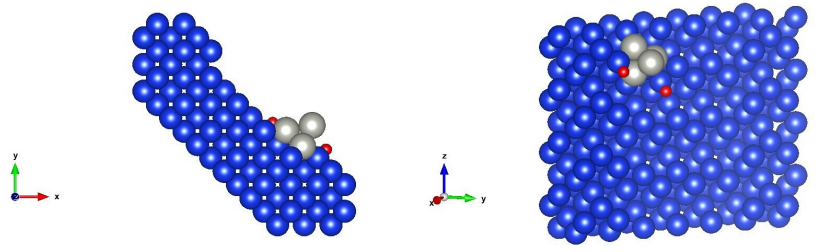

Fig. 6. After spillover from Pd cluster: split hydrogen forms bond with low valence (2) silicon located at nearby surface; top view (left) and side view (right).

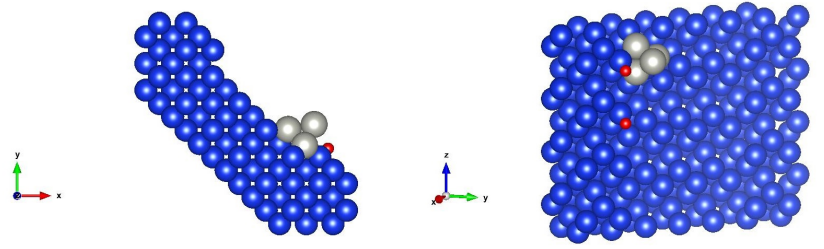

Fig. 7. After spillover from Pd cluster: split hydrogen forms bond with low valence (2) silicon located at alternate nearby surface locations; top view (left) and side view (right).

The spillover energy barrier was computed by comparing the ground state total energy of before and after spillover phases. The computed spillover energy barrier values for above configurations were 0.60 and $0.49 \mathrm{eV}$ respectively. Even though hydrogen atom has to overcome a lower spillover barrier of $0.49 \mathrm{eV}$ in second configuration, the dangling silicon atom is located almost 1 unit cell away from the Pd cluster which makes it less likely to occur. The more conservative choice is a resultant spillover barrier of $0.60 \mathrm{eV}$.

\section{Bond Hopping}

In the bond hopping process, the hydrogen atoms terminated in surface silicon atoms hop to the next available silicon atoms one atom further down the adjacent silicon unit cell along the z-direction. This corresponds to a bond 
hopping distance of $5.43 \AA$ A. Initially we considered bond hopping along $\mathrm{z}$ direction only. We kept the hydrogen atom fixed at the initial and final locations and then took 9 (nine) equidistant intermediate points in a straight line. We calculated ground state total energy for all those 11 locations, known as "images", using DFT. The difference in energy between the images plotted against the hopping distance resulted in following graph:

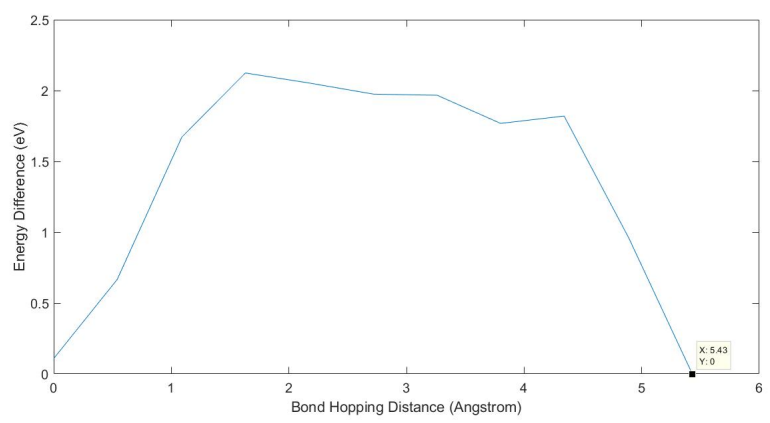

Fig. 8. Energy difference (eV) vs Bond Hopping Distance ( $\AA$ ) for bond hopping in a straight line along z-direction.

The peak of the energy barrier for z-direction hop of an entire unit cell is $1.63 \AA$ from the initial site. The hydrogen atom must overcome a substantial energy barrier of $2.12 \mathrm{eV}$ in order to bond hop to the next silicon cell. Next we explored alternate two-hop vectors whereby the hydrogen atom moves along diagonal $\mathrm{x}$ and $\mathrm{y}$ pathways. This movement is evaluated within a prismatic cylinder along the straight-line path having a radius of $0.10 \AA$ thus forming a cloud of possible locations for hydrogen atom. At least 10 points were investigated for each intermediate location giving a total of 120 DFT runs forming the whole 3D cloud. Ground state total energy was computed for all 120 points, which are plotted in figure 9. The size of the points increases with the increasing value of energy. Therefore, it is likely that the bond hopping will progress through a pathway connecting circles having smaller radii.

Next, we identified the lowest ground state energy of all cloud points. When we plotted the ground state total energy vs bond hopping distance for these points, the graph revealed one additional local minima as can be seen in figure 10 .

Even though the bond hopping energy barrier got reduced significantly from $2.12 \mathrm{eV}$ to $1.54 \mathrm{eV}$, but this doesn't represent the actual bond hopping pathway. But the presence of an additional local minima provided with the possibility of a quasi stable state between the initial and final image. In that case, the hydrogen atom may cover a hopping distance of $5.43 \AA$ in two serial hops having lower energy barrier compared to the straight line hop. It may hop from the initial state to the quasi stable state at a distance of around $2.50 \AA$ in a diagonal direction along the crystal face and finally the second hop from the quasi stable state to the final state at a distance of $2.93 \AA$.

In order to find the actual bond hopping pathway we utilized the Nudged Elastic Band (NEB) method. According

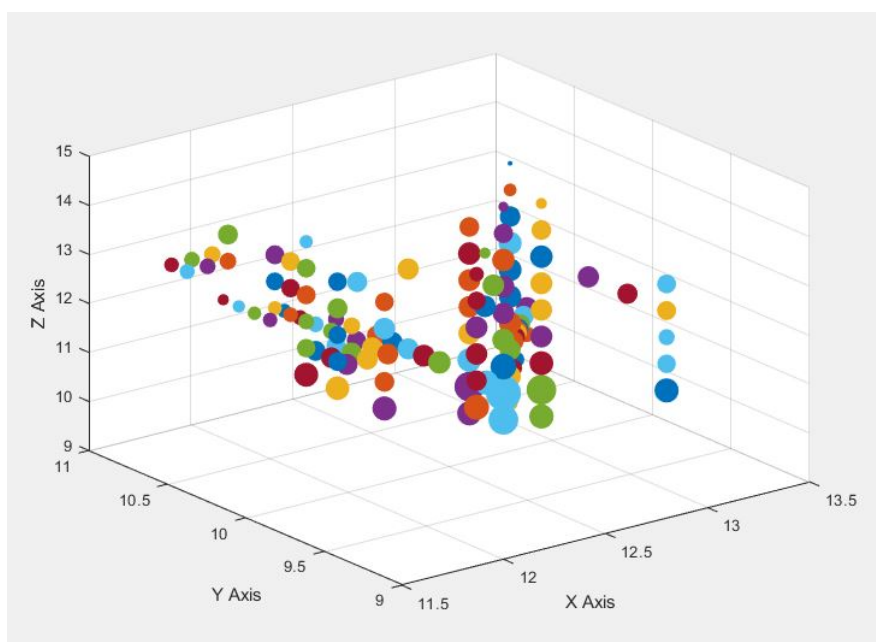

Fig. 9. 3D cloud of manually created possible location of $\mathrm{H}$ atom in bond hopping pathway

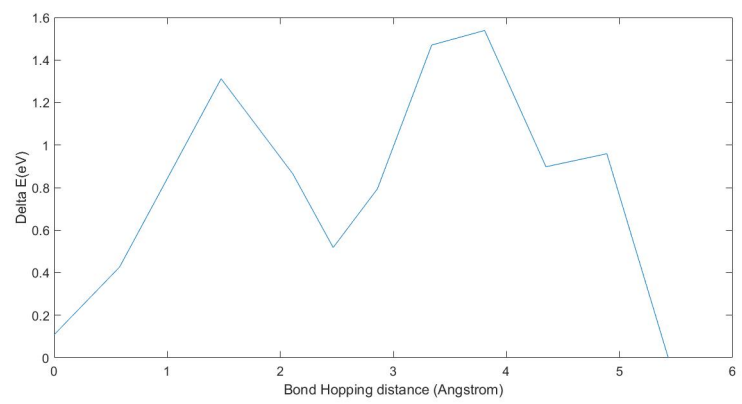

Fig. 10. Energy difference (eV) vs Bond Hopping Distance ( $\mathrm{A})$ calculated by taking minimum energy values for each point along $\mathrm{H}$ atom's bond hopping pathway from a cloud of manually created points in a 3D space.

to Jónsson et al. convergence to a minimum energy path is guaranteed in NEB method if a sufficient number of images are considered[13]. We initiated the NEB method by allowing the hydrogen atom to relax at the initial and final configurations ensuring two local minima. Improved tangent NEB method was used in our transition state investigation. ABINIT default tolerance value was used as the convergence criteria. Both single and double bond hopping pathways were considered. For alternate pathway consisting of two hops, the hopping distance changed to $4.50 \AA$ after hydrogen atom relaxation between which the first hop takes place. For the second hop, the distance is $2.35 \AA$. Therefore, we took 7 intermediate images for the first hop and 3 intermediate

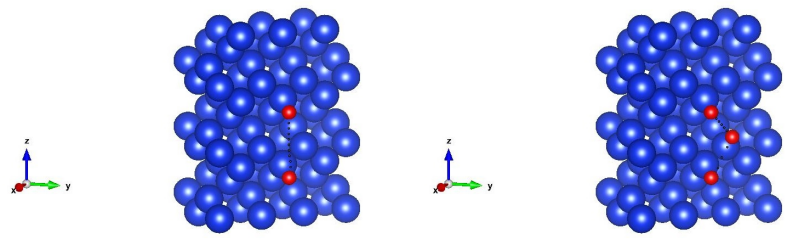

Fig. 11. Bond Hopping Alternate Pathways Consisting of Single Hop(left) and Double Hop(right). 
images for second hop. For direct hop, total number of images were kept at 11 as before. When this computation converged we got a direct hop bond hopping energy barrier of $2.06 \mathrm{eV}$. Whereas in alternate bond hopping pathway, the transition state gave an energy barrier of $1.74 \mathrm{eV}$ for the first hop. The energy barrier value is $1.45 \mathrm{eV}$ for the second hop. These values are smaller compared to the energy barrier of direct bond hop case. Therefore, we can conclude that hydrogen atom will preferentially diffuse along the silicon surface by bond hopping process consisting of two sequential diagonal hops along the crystal face in order to cover a vertical bond hopping distance of $5.43 \AA$

\section{E. Zero-Point Energy}

According to quantum mechanics, the lowest quantum mechanical energy for a collection of atoms will be:

$$
E=E_{0}+\frac{h \nu}{2}
$$

Here, $h$ is the plank's constant, $\nu$ is the classical vibrational frequency, and $E_{0}$ is the minimum energy computed using classical mechanics. The difference between this quantum mechanical energy and minimum energy according to classical mechanics gives the zero-point energy. Now if we apply this concept to a set of atoms, there will be zero-point energy contribution for each of the individual normal modes. The minimum energy considering all the normal modes is defined as:

$$
E=E_{0}+\sum_{i} \frac{h \nu_{i}}{2}
$$

The bond hopping energy barrier was computed using NEB method from classical mechanics perspective which doesn't include zero-point energy corrections. Since our system involves light atoms such as hydrogen, inclusion of zero-point energy correction may have significant impact on the bond hopping energy barrier[2]. In order to find zeropoint energy corrected activation energy, we will need to include zero-point energy corrections for both energy minima and transition state. In any set of atoms having $\mathrm{N}$ degrees of freedom, there will be $\mathrm{N}-1$ real vibrational modes and 1 (one) imaginary mode at transition state whereas the local minimum will have $\mathrm{N}$ real vibrational modes. The following equation gives zero-point energy corrected activation energy accordingly:

$$
\Delta E_{Z P}=\left(E^{\dagger}+\sum_{j=1}^{N-1} \frac{h \nu_{j}^{\dagger}}{2}\right)-\left(E_{A}+\sum_{i=1}^{N} \frac{h \nu_{i}}{2}\right)
$$

Here, $E^{\dagger}$ and $E_{A}$ represent ground state total energy at transition state and energy minima respectively. Their difference denotes the activation energy, $\Delta E$ defined in classical mechanics. In the above equation, $\nu_{i}$ and $\nu_{j}$ defines the normal mode frequencies at local minimum and transition states respectively. The above equation can be re-written as:

$$
\Delta E_{Z P}=\Delta E+\sum_{j=1}^{N-1} \frac{h \nu_{j}^{\dagger}}{2}-\sum_{i=1}^{N} \frac{h \nu_{i}}{2}
$$

Using the method defined in methods section, we calculated all these vibrational modes using ABINIT at both local minimum and the transition state. From equation (3) we get the frequency values: $\nu_{1}^{\dagger}=1.49 \times 10^{13} s^{-1}, \nu_{2}^{\dagger}=1.54 \times 10^{13}$ $s^{-1}$. Putting all these values in equation (10) for first hop we get,

$$
\Delta E_{Z P}=1.58 \mathrm{eV}
$$

This gives the zero-point corrected bond hopping energy barrier for the first hop. This value is $0.16 \mathrm{eV}$ lower than the bond hopping barrier calculated using classical mechanics. The zero-point correction for the second hop did not converge, and so no correction factor is included in the calculations below.

\section{F. Vibrational Frequency}

The vibrational frequency was calculated following the procedure depicted in subsection $\mathrm{C}$ within the Methods section. Multiple frequency values were computed for dissociation, spillover, and bond hopping. We consider the rate limiting frequency values for dissociation and spillover. The frequency values are $3.94 \times 10^{13} s^{-1}$ and $3.55 \times 10^{13} s^{-1}$ respectively. However, the direction of first bond hop closely aligns with z-coordinate whereas for second hop it is aligned with $\mathrm{x}$-coordinate. Therefore, we choose those frequency values that align with the corresponding directions. These values are $6.07 \times 10^{13} s^{-1}$ and $1.61 \times 10^{13} s^{-1}$ respectively.

\section{G. Surface Diffusion}

With the bond hop distance and the vibrational frequency it is now possible to compute the diffusion coefficient for surface movement of ensembles of hydrogen atoms on porous silicon. In the equation below $\mathrm{z}$ is the number of directions available to hop, $\alpha$ is the bond hop length in that direction, $\nu_{e}$ is the vibrational frequency associated with that particular direction, and $E_{a}$ is the activation energy which is the peak energy between start and end points for the hop.

$$
D=\frac{1}{z} \nu_{e} \alpha^{2} \exp \left(-\frac{E_{a}}{k T}\right)
$$

A tool for characterizing hydrogen carrying capacity is temperature programmed desorption (TPD). A study by Redhead [14] relates the peak temperature to the activation energy of absorption as a function of the rate of temperature increase $\beta$ and peak temperature $T_{p}$.

$$
E_{a}=R T_{p}\left[\ln \left(\frac{A T_{p}}{\beta}\right)-3.46\right]
$$

A summary of 15 experimental TPD runs is shown in Fig. 12 depicting a histogram of peak temperatures. Samples of as-synthesized hydrogenated nanoporous silicon exhibit a peak near to $376 \mathrm{C}$ with a standard deviation of 4.7. Samples to which Pd catalyst were deposited exhibited slightly lower peak temperatures, with the two around $360 \mathrm{C}$ being 3.3 standard deviations away from the average. This corresponds to a reduction of about $0.1 \mathrm{eV}$ in $E_{a}$.

Another TPD feature evident in the catalytically-modified samples is illustrated in Fig. 13 where short duration surges 


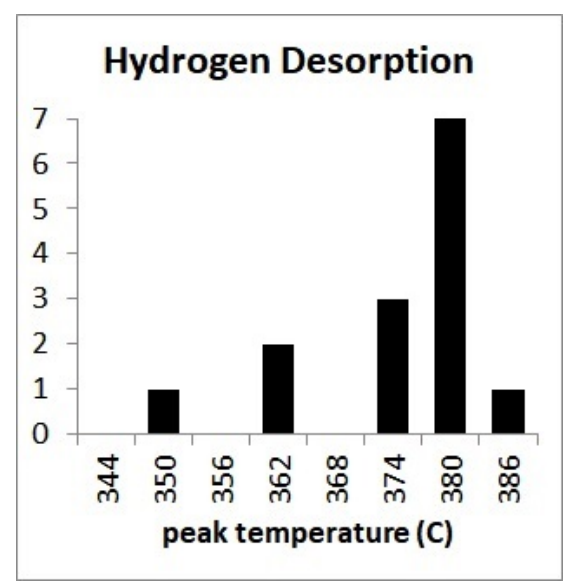

Fig. 12. Laboratory data from 14 runs of as-synthesized and catalyticallymodified porous silicon using temperature programmed desorption(TPD).

in hydrogen release are observed at temperatures lower than the peak. Samples without catalyst are smooth and lack these spikes.

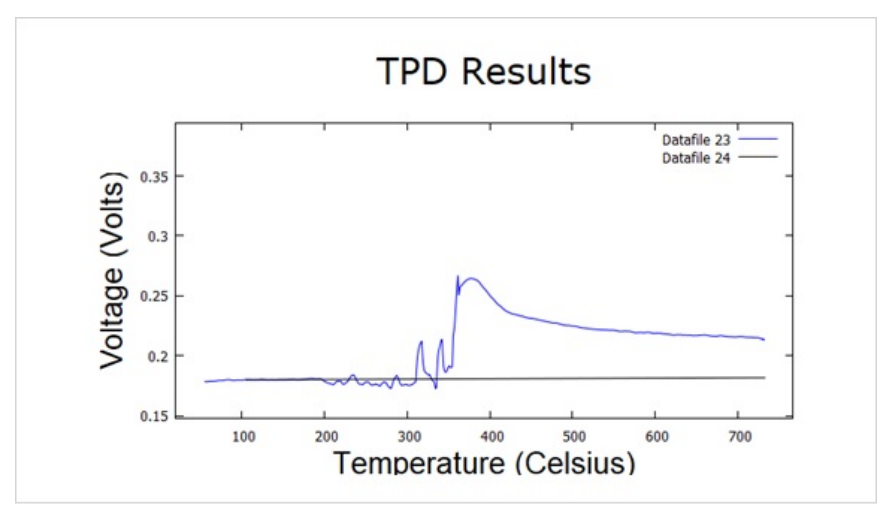

Fig. 13. Representative TPD trace (blue) showing lower-temperature release of hydrogen (spikes) plus regular peak.

\section{DISCUSSION}

Experimental results from TPD suggest that zero-point energy is not active in the surface diffusion of hydrogen on nanoporous silicon because the derived $E_{a}$ is $1.81 \mathrm{eV}$ which is just 3.8 percent greater than the DFT computed first hop energy of $1.74 \mathrm{eV}$. Because both hops are needed for longdistance movement of hydrogen atoms the higher energy barrier will dominate the transport. Computing diffusion coefficients from Eq. 11 yields values of diffusivity in the range of $10^{-16} \mathrm{~cm}^{2} / \mathrm{s}$ at $376 \mathrm{C}$ which is a very low value, comparable to hydrogen diffusion through metals. The evidence from Fig. 13 suggests that when catalyst clusters are nearby hydrogen storage sites the activation energy can be reduced. Using Eqs. (1) and (2) one obtains very high rates for dissociation and spillover. The limiting rate step is bond hopping. The $\mathrm{Si}-\mathrm{H}$ bond energy is sufficiently strong that bond hopping is a slow process at temperatures of practical interest. This consideration leads to a configuration with a much higher surface density of catalyst atoms, as shown in Fig. 14. Here, nearest neighbor silicon atoms with coordination number 2 and 3 are within reach of the spillover effect. If we also assume each cluster of four Pd atoms can hold $4 \mathrm{H}$ atoms, then an accounting of available vacant bonds gives 9.4 hydrogens per cluster. The surface density of Pd clusters on unit cell centers and face sites averages to eight $\mathrm{Pd}$ atoms per (100) unit cell area. The silicon matrix has 64 surface atoms, including 2 backbonds per unit cell area, and 240 bulk atoms, yielding a hydrogen gravimetric storage density of 1.36 percent (w/w). This calculated value is within the bounds of experimental data, which ranged from 1.3 to 2.3 percent $(\mathrm{w} / \mathrm{w})$ for catalyst-loaded $\mathrm{npSi}$. While this is a modest value for this metric, the benefit is seen in Fig. 15 which shows the recharge rate for the arrangement of Fig. 14. At temperatures commensurate with PEM fuel cell operation, and at pressures at or below that of a bicycle tire inflation, the fill time is on the order of 1.0 second. This further explains why our gaseous recharge analysis on laboratory samples did not detect a transient because the system response time was of a similar duration.

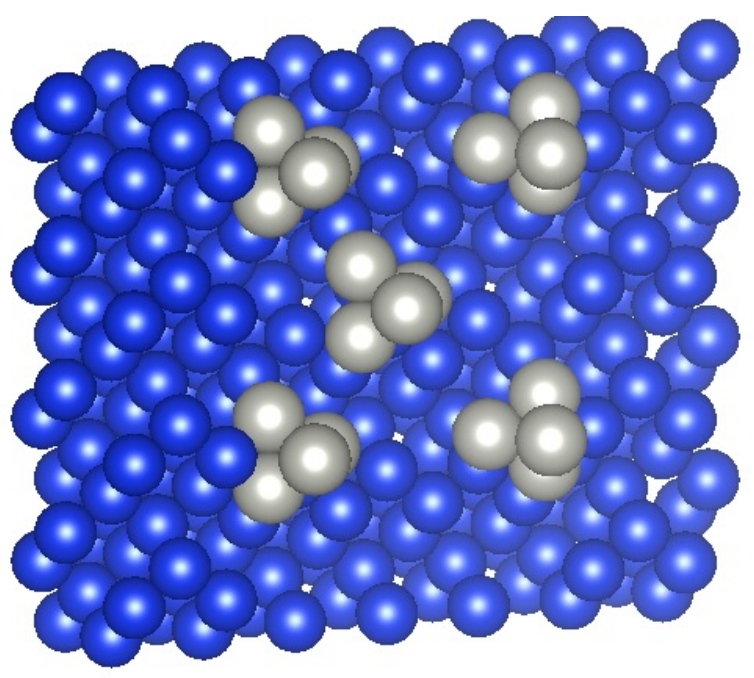

Fig. 14. Catalyst clustering scheme for fast recharge.

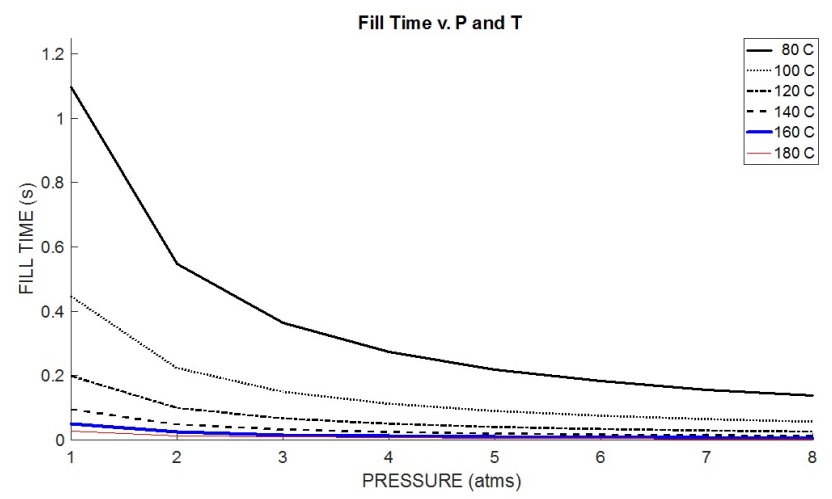

Fig. 15. Recharge dynamics versus pressure and temperature using configuration of Fig. 14 with spillover into nearest neighbors. 


\section{CONCLUSIONS}

The kinetics of recharging hydrogen gas onto catalyticallymodified nanoporous silicon (npSi) has been studied with a combination of Density Functional Theory (DFT) and laboratory testing. These results have been applied to the sequence of gaseous dissociation onto a catalyst cluster, spillover onto the npSi support or matrix, and bond-hopping along the interior surfaces of pores which have been created electrochemically within silicon crystal. The rate-limiting step is site-to-site diffusion along the silicon surface, which was discovered to proceed via two discrete hops per unit cell distance. One of the two inter-cell hops has a higher energy barrier, the magnitude of which was found to agree within 4 percent between DFT and lab measurements. To recharge catalytically-modified $\mathrm{npSi}$ as a hydrogen storage reservoir for practical applications requires that the surface density of catalyst clusters is high. Instant recharge is possible using temperatures available as waste heat from a PEM fuel cell or other modest heat source. The penalty is the cost and density of the catalyst. Future studies will explore non-PGM catalysts as the spillover energy of $0.60 \mathrm{eV}$ may be lower than required for practical applications. Calculations consistent with laboratory results have provided kinetic performance characterization of a novel means of hydrogen storage based.

\section{ACKNOWLEDGMENT}

This work was funded in part by NSF grant 1648748 .

\section{REFERENCES}

[1] P. Schubert and A. Wilks, "Thermodynamic analysis of a novel hydrogen storage material: Nanoporous silicon," Materials Innovations in an Emerging Hydrogen Economy, vol. 202, p. 105, 2009.

[2] D. Sholl and J. A. Steckel, Density functional theory: a practical introduction. John Wiley \& Sons, 2011.

[3] S. Banerjee, C. Pillai, and C. Majumder, "Dissociation and diusion of hydrogen on the mg (0001) surface: catalytic eect of $\mathrm{v}$ and ni double substitution," The Journal of Physical Chemistry C, vol. 113, no. 24, pp. 10574-10579, 2009.

[4] F. Bottin, S. Leroux, A. Knyazev, and G. Zerah, "Large-scale ab initio calculations based on three levels of parallelization," Computational Materials Science, vol. 42, no. 2, pp. 329-336, 2008.

[5] V. Lysenko, F. Bidault, S. Alekseev, V. Zaitsev, D. Barbier, C. Turpin, F. Geobaldo, P. Rivolo, and E. Garrone, "Study of porous silicon nanostructures as hydrogen reservoirs," The journal of physical chemistry B, vol. 109, no. 42, pp. 19711-19718, 2005.

[6] P. J. Schubert, "Solid-state hydrogen storage media and catalytic hydrogen recharging thereof," August 27 2013, US Patent 8,518,856.

[7] P. Schubert and A. Urbanek, "Hydrogen recharge dynamics and vessel design for porous silicon storage media," Proceedings of TechConnect World 2014, 2014.

[8] W. C. Conner Jr and J. L. Falconer, "Spillover in heterogeneous catalysis," Chemical reviews, vol. 95, no. 3, pp. 759-788, 1995.

[9] A. K. Singh, M. A. Ribas, and B. I. Yakobson, "H-spillover through the catalyst saturation: an ab initio thermodynamics study," Acs Nano, vol. 3, no. 7, pp. 1657-1662, 2009.

[10] C. Zhou, J. Wu, A. Nie, R. C. Forrey, A. Tachibana, and H. Cheng, ”On the sequential hydrogen dissociative chemisorption on small platinum clusters: a density functional theory study," The Journal of Physical Chemistry C, vol. 111, no. 34, pp. 12773-12778, 2007.

[11] A. D. Lueking and R. T. Yang, "Hydrogen spillover to enhance hydrogen storagestudy of the eect of carbon physicochemical properties," Applied Catalysis A: General, vol. 265, no. 2, pp. 259-268, 2004.

[12] J. Moc, D. G. Musaev, and K. Morokuma, "Adsorption of multiple h2 molecules on pd 3 and pd4 clusters. a density functional study," The Journal of Physical Chemistry A, vol. 104, no. 49, pp. 11606-11614, 2000 .
[13] H. Jónsson, G. Mills, and K. W. Jacobsen, "Nudged elastic band method for finding minimum energy paths of transitions," in Classical and quantum dynamics in condensed phase simulations. World Scientific, 1998, pp. 385-404.

[14] P. A. Redhead, "Thermal Desorption of Gases," Vacuum, 12, 203-211 (1962). 